\title{
Comportamiento de la diversidad arbórea-arbustiva en zonas de pastoreo en un área natural protegida del trópico seco poblano
}

\section{Behaviour of the tree-bush diversity in grazing zones in a natural protected area of the poblano dry tropic}

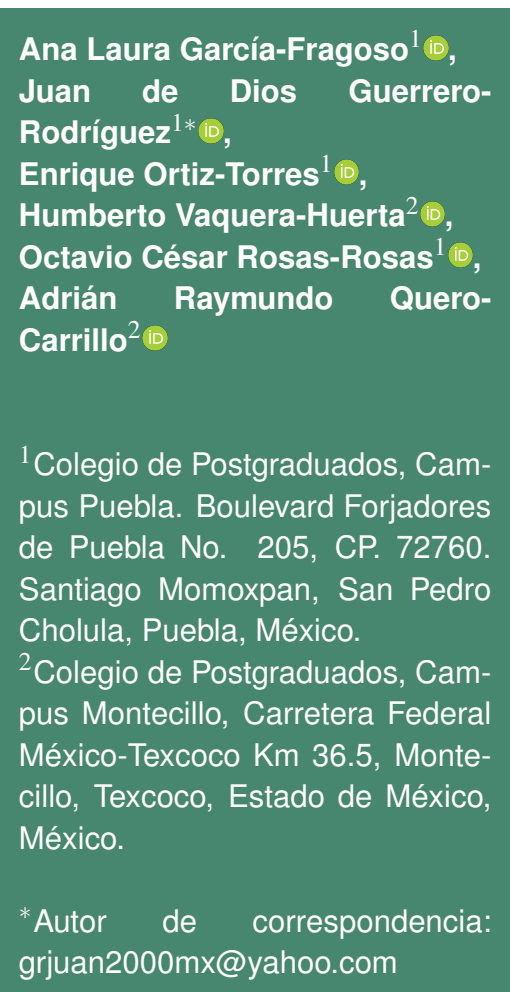

Artículo científico

Recibido: 24 de febrero 2021

Aceptado: 13 de septiembre 2021

Como citar: García-Fragoso AL, Guerrero-Rodríguez JD, OrtizTorres E, Vaquera-Huerta $\mathrm{H}$, Rosas-Rosas OC, Quero-Carrillo AR (2021) Comportamiento de la diversidad arbórea-arbustiva en zonas de pastoreo en un área natural protegida del trópico seco poblano. Ecosistemas y Recursos Agropecuarios Núm. Esp. II: e2879. DOI: 10.19136/era.a8nll.2879
RESUMEN. La selva baja caducifolia tiene alta diversidad y riqueza biológica, que brinda varios servicios ambientales; no obstante, en los últimos años, algunas actividades humanas sin control han contribuido a su degradación y pérdida. Este es un ecosistema propio de la Reserva Estatal Sierra del Tentzo, en el estado de Puebla, México, donde el uso de recursos naturales es fuente de ingreso y sustento de sus habitantes. Dado que existe controversia sobre la afectación de la ganadería por el pastoreo, el objetivo del presente estudio fue determinar la diversidad de especies arbóreas y arbustivas en zonas con y sin pastoreo. Se muestrearon árboles y arbustos de altura mayor o igual a un metro, en los municipios de Huehuetlán El Grande y Huaquechula, donde se establecieron 300 cuadrantes para cada zona y se tomaron muestras de cada especie. Mediante el estimador no paramétrico Chao 2 y el índice Shannon-Wiener ( $\mathrm{H}^{\prime}$ ) se determinó diversidad, dominancia y equidad, obteniéndose la riqueza con curvas de acumulación de especies. Los valores de H' para las dos zonas fueron altos, pero varias especies son de sucesión secundaria e indicadoras de disturbio, sugiriendo que, el pastoreo y las actividades que ahí se realizan impactan de manera importante en la estructura y funcionalidad de la diversidad arbóreo-arbustiva nativa.

Palabras clave: Área natural protegida, árboles y arbustos, Índices ecológicos, selva baja caducifolia.

ABSTRACT. The tropical dry forest is characterized by its high diversity and biological richness, which provides several environmental services; nonetheless, in recent years, some uncontrolled human activities contribute to its degradation and loss. This is an ecosystem of the Sierra del Tentzo State Reserve, in the State of Puebla, México, where the use of natural resources is the main source of income and livelihood of its inhabitants. Since there is controversy about the effect of grazing, the present study was proposed, whose objective was to determine the diversity of arboreal species in areas with and without grazing. A sampling of trees and shrubs with a height greater than or equal to one meter was carried out in the municipalities of Huehuetlán El Grande and Huaquechula, establishing 300 quadrants for each zone, where samples of each species were taken. Using the non-parametric Chao 2 estimator and the Shannon-Wiener index ( $\left.H^{\prime}\right)$ diversity, dominance and equity were determined, while species richness was obtained with accumulation curves. The values of $\mathrm{H}^{\prime}$ for the areas with and without grazing were high, but several of the species reported are of secondary succession, an indicative of disturbance, showing that grazing and the activities carried out there are having an important impact on the structure and functionality of the native arboreal-shrub diversity.

Key words: Protected area, trees and shrubs, ecological indices, tropical dry forest. 


\section{INTRODUCCIÓN}

La selva baja caducifolia (SBC) comprende el $42 \%$ de los ecosistemas forestales del mundo (Kalacska et al. 2004) y es de los lugares donde la tasa de conversión de selvas a tierras agrícolas o a tierras de pastoreo es mayor que en cualquier otro tipo de vegetación (Trejo y Dirzo 2000, Kauffman et al. 2003). Algunos investigadores (Gallardo-Cruz et al. 2005, García-Romero et al. 2005) afirman que todavía se desconoce gran parte de las funciones y procesos ecológicos que alberga en su interior este tipo de selvas, así como las características de su distribución y abundancia, pues sigue siendo de las menos investigadas y más amenazadas por las actividades humanas (Gallardo-Cruz et al. 2005, Casanova-Lugo et al. 2014).

En este tipo de selva, la ganadería, que es una actividad económica importante de subsistencia para las familias campesinas, ha aumentado considerablemente; para lo cual, en muchos casos, se desmonta (Baraza y Valiente-Banuet 2012), y se propicia el libre pastoreo en los agostaderos o en praderas inducidas. Algunos autores consideran que después del fuego, el pastoreo es el que más propicia la alteración, degradación, pérdida de área y biomasa de los ecosistemas naturales del mundo (Müller et al. 2011, Villanueva-Partida et al. 2019). La SBC está presente en la Reserva Estatal Sierra del Tentzo (REST) en el estado de Puebla, México y en ella se practican diferentes actividades agropecuarias como el pastoreo, la recolección de leña, plantas medicinales, forraje, frutos, entre otros; aprovechamientos comunes en otras partes del mundo (Nautiyal y Kaechele 2006, Vedeld et al. 2012). En dicha Reserva, de manera supuesta, se le atribuye a la ganadería un efecto negativo en la riqueza y abundancia florística (SMADSOT 2011), aspecto que algunos autores (Díaz et al. 2007, Müller et al. 2011) han observado en algunas otras partes del mundo. Sin embargo, hasta la fecha no existe un estudio que analice y provea información sobre los posibles impactos causados por la ganadería en la SBC de la REST.
Existen investigaciones con resultados contrastantes en cuanto a los impactos causados a la selva por el pastoreo. Según Belsky y Blumenthal (1997) y Hernández et al. (2000), el pastoreo por ganado altera la dinámica de los bosques y selvas naturales, ya que contribuye a la disminución de la biomasa y densidad de herbáceas, propicia también el desplazamiento de especies palatables por otras no palatables y, pueden desaparecer especies nativas (Kauffman et al. 2003). Al respecto, Williams-Linera y Lorea (2009), mencionan que en los senderos y sitios pastoreados existe menor riqueza que en los sitios de mayor altitud y pendiente en selvas caducifolias. No obstante, otros autores mencionan que, con la ganadería, se puede contribuir a la mejora y recuperación de ecosistemas (Bermejo et al. 2012, García et al. 2012, Benthien et al. 2018). En algunos casos se ha dicho que complementan los patrones de consumo, principalmente con combinación de especies animales; también, al darse el ramoneo se favorece el control de especies indeseables ya que actúa como control biológico o se estimula el mantenimiento de los pastizales al reducirse la sombra por árboles y arbustos. Adicionalmente, los rumiantes contribuyen a la dispersión de semillas viables, en las heces (Baraza y ValienteBanuet 2008), lo que modifica la presencia y distribución de ciertas especies vegetales. Por tanto, existe evidencia que el pastoreo puede modificar la diversidad de plantas negativa o positivamente. Pero ciertas consideraciones se deben de tomar en cuenta, pues como mencionan Arévalo et al. (2011) y Bermejo et al. (2012), los efectos del pastoreo sobre los diferentes ambientes pueden ser inciertos o inestables, por lo que es necesario realizar estudios específicos de cada ecosistema, ya que no se puede generalizar un mismo efecto para todos los ambientes. Por lo anterior, el objetivo de esta investigación fue evaluar la diversidad de especies arbóreas y arbustivas en zonas pastoreadas y no pastoreadas en la selva baja caducifolia en transición a bosque de encino de la REST. 


\section{MATERIALES Y MÉTODOS}

\section{Zona de estudio}

La REST se ubica en la parte centro oeste del estado de Puebla, México, en los paralelos $18^{\circ}$ $42^{\prime} 26^{\prime \prime}$ Norte y en los meridianos $98^{\circ} 26^{\prime} 30^{\prime \prime}$ Oeste, a una altitud de $1500 \mathrm{msnm}$ y tiene una superficie de 57815.3 ha (SSAOT 2011a, 2011b). En el decreto estatal de su formación se estableció que la ganadería se fomente de manera racional en los ejidos y tierras comunales. En ella, la selva baja caducifolia representa el 20.7\% (12 603.2 ha). Los climas que existen en dicha vegetación son el cálido (Aw), semicálido (Cw) y semiseco (BS) con lluvias en verano (García 2004). La temperatura es superior a los $22{ }^{\circ} \mathrm{C}$ y tiene una precipitación anual que va de los 800 a 1000 mm, con alto índice de evapotranspiración (Fernández-Nava et al. 1998). Los suelos predominantes en esta área son rendzina, regosol y litosol (SEMARNAP 1998). Este estudio se realizó en los ejidos y tierras comunales de los municipios Huehuetlán El Grande y Huaquechula, los cuales presentan gran porcentaje de selva baja, además de que en estos municipios la ganadería es una práctica económica importante (INEGI 2017).

\section{Unidades de estudio}

Se consideraron zonas con pastoreo por más de 20 años (cercanas a veredas de uno a cinco metros y a las comunidades desde donde iniciaba la selva) y zonas sin pastoreo (alejadas de las mismas comunidades en estudio y donde la posibilidad de pastoreo es muy difícil o nula). El pastoreo, es en mayor proporción de caprinos y bovinos, y en menor medida por ovinos y animales de trabajo (equinos). El tamaño de los hatos varía entre 35 y 76 animales y la duración del pastoreo en promedio es de nueve horas al día, y se recorren distancias de entre 3 y $5 \mathrm{~km}$. Para determinar las unidades de estudio, el muestreo se llevó a cabo en compañía de ganaderos, autoridades ejidales y jueces de paz de las distintas comunidades, quienes determinaron las zonas donde se efectuaba y no se efectuaba el pastoreo. En ambos escenarios se muestreó a través de cuadrantes de 20x20 m hechos de cuerdas marcadas. El muestreo de la vegetación se realizó sólo para el estrato arbóreo y arbustivo (especies leñosas) con una altura mayor o igual a un metro.

En cada condición de estudio se establecieron un total de 300 cuadrantes (12 ha), distribuidos en lugares representativos de la selva baja con afinidad a bosque de encino, en las tierras comunales y ejidales de los municipios mencionados.

\section{Composición de especies}

Las especies vegetales se identificaron botánicamente con base al cotejo de plantas, estructuras reproductivas y vegetativas colectadas durante la estación de verano y con ayuda de ejemplares impresos (Huerta-Zamacona et al. 2009, RodríguezAcosta et al. 2009) y bases especializadas como worldfloraonline para elaborar el listado florístico.

\section{Riqueza y diversidad}

El análisis de diversidad por condición de estudio se basó en la riqueza de especies (S). Se elaboraron matrices de especies para determinar la riqueza y diversidad proporcional de las especies en las zonas con y sin pastoreo. El análisis se realizó en el programa EstimateS, versión 8.2 (Colwell 2005). Se comparó la riqueza de especies entre las dos condiciones de muestreo utilizando curvas de acumulación de especies y el cálculo de los estimadores no paramétricos cobertura de abundancia (ACE), Chao 1 y Chao 2 (Chao et al. 2005). Las curvas de acumulación de especies estiman el número de especies esperadas a partir de un muestreo, donde a medida que aumentan las muestras, el número de especies se va acumulando (Colwell et al. 2004). Chao 2 estima el número de especies esperadas considerando la relación entre el número especies únicas y el número de especies duplicadas (Chao et al. 2005, Villarreal et al. 2006). Las curvas de acumulación de especies se produjeron mediante el reordenamiento aleatorio repetido (1000 repeticiones) (Cavieres y Badano 2009).

Se realizó un comparativo de la composición entre sitios con y sin pastoreo mediante un análisis de similitud (ANOSIM) para corroborar la contribución de especies al porcentaje de disimilitud utilizando la 
subrutina SIMPER con el programa PAST versión 4.3 (Hammer et al. 2001). El ANOSIM fue de una vía con permutación de 9999, y un nivel de significancia $p<$ 0.05 .

Para determinar la diversidad alfa se emplearon los siguientes índices:

Índice de Shannon-Wiener ( $\left.H^{\prime}\right)$ : con él se mide el grado de incertidumbre en predecir a qué especie pertenecerá un individuo elegido al azar de una superficie muestreada. Este índice asume que todas las especies están representadas en las muestras y son seleccionados al azar, e indica qué tan uniformes están representadas las especies (en abundancia) teniendo en cuenta todas las especies muestreadas (Pla 2006, Villarreal et al. 2006). Adquiere valores entre cero, cuando existe una sola especie, y el logaritmo del número de especies (S), cuando todas las especies están representadas por el mismo número de individuos (Magurran 1988). Este índice se calculó con la siguiente fórmula:

$$
H^{\prime}=-\sum_{i=l}^{s} P i \ln P i
$$

Dónde: $H^{\prime}=$ Índice de Shannon-Wiener; $\mathrm{Pi}=$ Proporción del número de individuos de la especie i con respecto al total; In = Logaritmo natural. Así mismo, con base en este índice se realizó una prueba de "t" modificada por Hutcheson utilizando el paquete $\mathrm{R}$ versión 4.02.

Dominancia: indica cuan desigual están distribuidas las especies en una comunidad; su valor oscila entre 0 y 1 , calculándose por la división $\frac{N i}{N}$. Dónde: $\mathrm{Ni}=$ Número de individuos de la especie más abundante en la muestra; y $\mathrm{N}=$ número total de individuos en la muestra.

Índice de Equidad (E): emplea el índice de Shannon $\left(\mathrm{H}^{\prime}\right)$ y sus valores van de 0 a 1 , dónde 0 indica la mínima equidad (o que sólo una especie está presente en el área de muestreo) y 1 que todas las especies tienen la misma abundancia (Magurran 1988). Su cálculo con la siguiente fórmula:

$$
E=\frac{H^{\prime}}{\ln S}
$$

Dónde: H' = índice de Shannon; ln = logaritmo natural (o base e); y $\mathrm{S}=$ número de especies en la muestra.

\section{RESULTADOS}

Se encontraron 36 familias, 93 géneros (Figura 1) y 131 especies. Del total de especies, 113 se ubicaron en la zona sin pastoreo y 120 en la zona con pastoreo, compartiendo ambas zonas 102 especies (Tabla 1). De las especies encontradas, 112 fueron identificadas a nivel de especie (85.5\%), ocho a nivel de género $(6.1 \%)$ y 11 especies quedaron identificadas a nivel familia (8.4\%). La distribución de especies por familia (Figura 1) fue desigual; la familia más representativa fue la Fabaceae con 31 especies (23.48\%).

El valor de dominancia para la zona con pastoreo fue de 0.107 ; donde la especie más dominante fue Opuntia depressa Rose de la familia Cactaceae; para la zona sin pastoreo el valor de dominancia fue de 0.091 y la especie más dominante fue Viguiera insignis Miranda. De acuerdo a la prueba de ANOSIM, se encontraron diferencias significativas entre ambas zonas $(p=0.0001, R=0.06)$, con 13 especies acumulando el $51 \%$ de la disimilitud (Tabla 1); de entre ellas, Brahea dulcis (Kunth) Mart., Opuntia depressa Rose, Dodonaea viscosa (L.) Jacq, Lippia graveolens Kunth y Acacia bilimekii J. F. Macbr. tuvieron la mayor abundancia media en la zona con pastoreo que en la de sin pastoreo, mientras que, en esta última, las especies Viguiera insignis Miranda y Mimosa polyantha Benth. fueron las más abundantes. En global, en 67 especies hubo mayor número de individuos en la zona con pastoreo; mientras en la zona sin pastoreo hubo 52 especies con mayor número de individuos que en la zona con pastoreo y 12 especies que no se aparecieron en la zona con pastoreo.

El valor de equidad en la zona de pastoreo fue de 0.776 , mientras que la zona sin pastoreo tuvo un valor de 0.82 , mayor que el de las zonas pastoreadas. De acuerdo a la Prueba de "t" modificada por Hutcheson, las zonas difirieron $(p<0.0001)$ en diversidad, teniendo la zona sin pastoreo un valor de Índice de Shannon de 2.14 y la zona con pastoreo de 1.95 (Figura 2). Sin el ajuste de Hutcheson, el valor del Índice de Shannon-Wiener (H') obtenido 


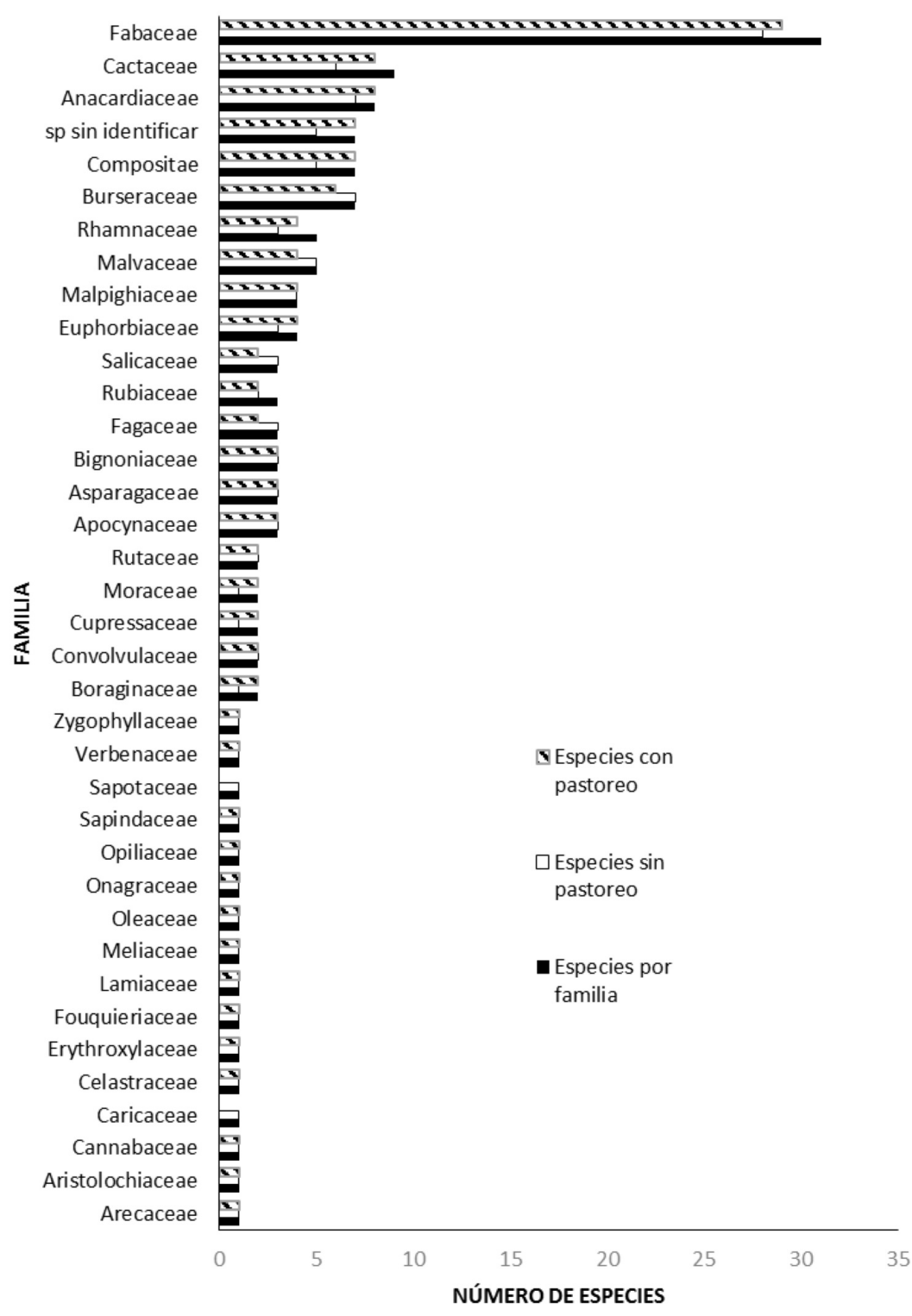

Figura 1. Distribución de especies en las familias registradas en la selva baja caducifolia de la Reserva Estatal Sierra del Tentzo (total y por condición de con y sin pastoreo).

para la zona con pastoreo fue de 3.72 y para la zona sin pastoreo fue de 3.88 .

Las curvas de acumulación de especies (Figura 3) para las zonas pastoreadas y sin pastoreo, tuvieron una asíntota. El estimador no paramétrico de Chao 2 a partir de 1000 remuestreos realizados al máximo número de muestras $(\mathrm{N})$, mostró que con el $95 \%$ del intervalo de confianza se solapa con el valor máximo de riqueza de especies con una exactitud de 0.979 y 0.986 para las zonas sin pastoreo y con pastoreo, respectivamente. Por su parte, el estimador ACE 1 tuvo exactitudes de 0.985 y 0.994 para las zonas sin pastoreo y con pastoreo, respectivamente. Chao 1 tuvo exactitudes de 0.991 y 0.998 para las zonas sin pastoreo y con pastoreo, respectivamente. 
Tabla 1. Número de individuos por especie en cada familia en la zona de pastoreo (CP) y sin pastoreo (SP).

\begin{tabular}{|c|c|c|c|c|c|c|c|c|c|}
\hline \multirow[b]{2}{*}{ Familia y especies } & \multirow[b]{2}{*}{$\begin{array}{l}\text { Forma } \\
\text { de vida }\end{array}$} & \multirow[b]{2}{*}{$\mathrm{SP}$} & \multirow[b]{2}{*}{$\begin{array}{l}\mathrm{EE} \\
\mathrm{SP}\end{array}$} & \multirow[b]{2}{*}{$\mathrm{CP}$} & \multirow[b]{2}{*}{$\begin{array}{l}\mathrm{EE} \\
\mathrm{CP}\end{array}$} & \multicolumn{4}{|c|}{ SIMPER } \\
\hline & & & & & & $\begin{array}{l}\text { Disimilitud } \\
\text { promedio }\end{array}$ & $\begin{array}{r}\text { Contribución } \\
(\%)\end{array}$ & $\begin{array}{r}\text { Abundancia } \\
\text { media SP }\end{array}$ & $\begin{array}{r}\text { Abundancia } \\
\text { media } \mathrm{CP}\end{array}$ \\
\hline Familia Anacardiaceae & & & & & & & & & \\
\hline Comocladia engleriana Loes. & Árbol * & 239 & 0.098 & 258 & 0.115 & 1.19 & 1.346 & 0.797 & 0.86 \\
\hline Actinocheita potentillifolia (Turcz.) Bullock & Árbol ${ }^{\star}$ & 256 & 0.119 & 62 & 0.041 & 0.8085 & 0.9145 & 0.853 & 0.207 \\
\hline Pistacia mexicana & Árbol $^{\star}$ & 129 & 0.081 & 85 & 0.074 & 0.6596 & 0.7461 & 0.43 & 0.283 \\
\hline Rhus standleyi F.A. Barkley & 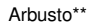 & 119 & 0.118 & 50 & 0.042 & 0.3973 & 0.4494 & 0.397 & 0.167 \\
\hline Rhus chondroloma Standl. & Arbusto** & 69 & 0.061 & 77 & 0.053 & 0.3536 & 0.4 & 0.23 & 0.257 \\
\hline Spondias mombin $\mathrm{L}$. & Árbol** & 24 & 0.022 & 61 & 0.047 & 0.2451 & 0.2772 & 0.08 & 0.203 \\
\hline Amphipterygium adstringens (Schltdl.) Standl. & Árbol ${ }^{\star}$ & 17 & 0.018 & 50 & 0.040 & 0.1718 & 0.1944 & 0.0567 & 0.167 \\
\hline $\begin{array}{l}\text { Cyrtocarpa procera Kunth } \\
\text { Familia Apocynaceae }\end{array}$ & Árbol ${ }^{*}$ & - & 0.000 & 17 & 0.017 & 0.0696 & 0.0788 & 0 & 0.0567 \\
\hline Plumeria rubra L. & Árbol** & 295 & 0.130 & 39 & 0.034 & 1.079 & 1.22 & 0.983 & 0.13 \\
\hline Cascabela thevetia (L.) Lippold & Árbol** & 124 & 0.060 & 95 & 0.058 & 0.5852 & 0.6619 & 0.413 & 0.317 \\
\hline $\begin{array}{l}\text { Tabernaemontana grandiflora Jacq. } \\
\text { Familia Arecaceae }\end{array}$ & Árbol ${ }^{\star}$ & 21 & 0.023 & 36 & 0.050 & 0.2168 & 0.2452 & 0.07 & 0.12 \\
\hline $\begin{array}{l}\text { Brahea dulcis (Kunth) Mart. } \\
\text { Familia Aristolochiaceae }\end{array}$ & Arbusto** & 1045 & 0.357 & 1862 & 0.536 & 6.252 & 7.072 & 3.48 & 6.21 \\
\hline $\begin{array}{l}\text { Asclepias curassavica L. } \\
\text { Familia Asparagaceae }\end{array}$ & Arbusto* & 83 & 0.067 & 16 & 0.036 & 0.265 & 0.2997 & 0.277 & 0.0533 \\
\hline Yuccasp. & Arbusto* & 199 & 0.089 & 123 & 0.061 & 0.8193 & 0.9267 & 0.663 & 0.41 \\
\hline Dasylirion spp. & Arbusto* & 7 & 0.011 & 63 & 0.063 & 0.1774 & 0.2007 & 0.0233 & 0.21 \\
\hline Beaucarnea gracilis Lem. & Arbusto* & 4 & 0.011 & 26 & 0.043 & 0.0604 & 0.0683 & 0.0133 & 0.0867 \\
\hline Familia Bignoniaceae & & & & & & & & & \\
\hline Tecoma stans (L.) Juss. Ex Kunth & Arbusto $^{\star \star}$ & 83 & 0.049 & 248 & 0.137 & 0.8746 & 0.9892 & 0.277 & 0.827 \\
\hline Tabebuia rosea (Bertol.) Bertero ex A.DC. & Árbol $^{\star}$ & 43 & 0.037 & 6 & 0.014 & 0.1848 & 0.209 & 0.143 & 0.02 \\
\hline $\begin{array}{l}\text { Astianthus viminalis (Kunth) Baill. } \\
\text { Familia Boraginaceae }\end{array}$ & Arbusto* & 7 & 0.012 & 15 & 0.017 & 0.0744 & 0.0842 & 0.0233 & 0.05 \\
\hline Tournefortia hirsutissima L. & Arbusto** & - & 0.000 & 4 & 0.013 & 0.0228 & 0.0258 & 0 & 0.0133 \\
\hline $\begin{array}{l}\text { Cordia morelosana Standl. } \\
\text { Familia Burseraceae }\end{array}$ & Árbol ${ }^{\star \star}$ & 2 & 0.005 & 2 & 0.005 & 0.0168 & 0.019 & 0.0067 & 0.0067 \\
\hline Bursera simaruba (L.) Sarg. & Árbol ${ }^{*}$ & 341 & 0.147 & 147 & 0.072 & 1.333 & 1.508 & 1.14 & 0.49 \\
\hline Bursera fagaroides (Kunth) Engl. & Árbol $^{\star}$ & 271 & 0.094 & 144 & 0.084 & 1.052 & 1.189 & 0.903 & 0.48 \\
\hline Bursera glabrifolia (Kunth) Engl. & Árbol ${ }^{\star}$ & 120 & 0.061 & 189 & 0.113 & 0.8292 & 0.9379 & 0.4 & 0.63 \\
\hline Bursera copallifera (Sessé \& Moc. ex DC.) Bullock & Árbol $^{*}$ & 75 & 0.042 & 47 & 0.044 & 0.3473 & 0.3928 & 0.25 & 0.157 \\
\hline Bursera morelensis Ramírez & Árbol* & 37 & 0.036 & 10 & 0.011 & 0.1325 & 0.1499 & 0.123 & 0.0333 \\
\hline Bursera aptera Ramírez & Árbol $^{\star}$ & 1 & 0.003 & 37 & 0.037 & 0.1057 & 0.1196 & 0.0033 & 0.123 \\
\hline $\begin{array}{l}\text { Bursera microphylla A.Gray } \\
\text { Familia Cactaceae }\end{array}$ & Árbol* & 1 & 0.003 & - & 0.000 & 0.0023 & 0.0026 & 0.0033 & 0 \\
\hline Opuntia depressa Rose & Arbusto** & 869 & 0.300 & 2026 & 0.514 & 6.13 & 6.933 & 2.9 & 6.75 \\
\hline Neobuxbaumia mezcalaensis Bravo & Arbusto* & 69 & 0.088 & 84 & 0.102 & 0.498 & 0.5633 & 0.23 & 0.28 \\
\hline Nopalea cochenillifera(L.) Salm-Dyck & Arbusto** & 51 & 0.061 & 7 & 0.010 & 0.1843 & 0.2084 & 0.17 & 0.0233 \\
\hline Pachycereus marginatus (DC.) Britton \& Rose & Arbusto* $^{\star}$ & 2 & 0.005 & 36 & 0.049 & 0.1243 & 0.1405 & 0.0067 & 0.12 \\
\hline Lemaireocereus weberi (J.M.Coult.) Britton \& Rose & Arbusto* & 2 & 0.007 & 13 & 0.022 & 0.0558 & 0.0631 & 0.0067 & 0.0433 \\
\hline Stenocereus pruinosus (Otto ex Pfeiff.) Buxb. & Arbusto* & - & 0.000 & 18 & 0.031 & 0.0538 & 0.0609 & 0 & 0.06 \\
\hline Myrtillocactus geometrizans (Mart. ex Pfeiff) Console & Arbusto* & - & 0.000 & 5 & 0.009 & 0.0175 & 0.0198 & 0 & 0.0167 \\
\hline Pachycereus weberi (J.M. Coult.) Backeb & Arbusto* & - & 0.000 & 2 & 0.005 & 0.0084 & 0.0095 & 0 & 0.0067 \\
\hline $\begin{array}{l}\text { Stenocereus stellatus (Pfeiff.) Riccob. } \\
\text { Familia Cannabaceae }\end{array}$ & Arbusto* & 2 & 0.007 & - & 0.000 & 0.0066 & 0.0075 & 0.0067 & 0 \\
\hline $\begin{array}{l}\text { Celtis caudata Planch. } \\
\text { Familia Caricaceae }\end{array}$ & Arbusto* & 8 & 0.010 & 13 & 0.014 & 0.06 & 0.0679 & 0.0267 & 0.0433 \\
\hline $\begin{array}{l}\text { Carica papaya L. } \\
\text { Familia Celastraceae }\end{array}$ & Arbusto* & 4 & 0.013 & - & 0.000 & 0.014 & 0.0158 & 0.0133 & 0 \\
\hline $\begin{array}{l}\text { Wimmeria microphylla Radlk. } \\
\text { Familia Compositae }\end{array}$ & Arbusto* & 185 & 0.119 & 141 & 0.092 & 0.915 & 1.035 & 0.617 & 0.47 \\
\hline Viguiera insignis Miranda & Arbusto** & 1437 & 0.622 & 416 & 0.251 & 4.516 & 5.107 & 4.79 & 1.39 \\
\hline Montanoa tomentosa (Cerv.) DC. & Arbusto** & 344 & 0.172 & 208 & 0.134 & 1.406 & 1.59 & 1.15 & 0.693 \\
\hline Viguiera eriophora var. poblana (Panero \& E.E.Schill.) B.L.Turner & Arbusto*^ & 292 & 0.219 & 28 & 0.054 & 0.9013 & 1.019 & 0.973 & 0.0933 \\
\hline Senecio praecox (Cav.) DC. & Arbusto* $^{\star}$ & 90 & 0.056 & 133 & 0.114 & 0.5847 & 0.6613 & 0.3 & 0.443 \\
\hline Gochnatia hypoleuca (DC.) A.Gray & Arbusto* & - & 0.000 & 60 & 0.137 & 0.1668 & 0.1887 & 0 & 0.2 \\
\hline Sp. 1 & 25 & 0.064 & 2 & 0.007 & 0.05 & 0.0565 & 0.0833 & 0.0067 & \\
\hline Otopappus imbricatus (Sch.Bip.) S.F.Blake & Arbusto** & - & 0.000 & 10 & 0.030 & 0.026 & 0.0294 & 0 & 0.0333 \\
\hline $\begin{array}{l}\text { Familia Convolvulaceae } \\
\text { Ipomoea wolcottiana Rose }\end{array}$ & Árbol** & 252 & 0.118 & 565 & 0.239 & 2.182 & 2.468 & 0.84 & 1.88 \\
\hline $\begin{array}{l}\text { Ipomoea murucoides Roem. \& Schult. } \\
\text { Familia Cupressaceae }\end{array}$ & Árbol ${ }^{\star \star}$ & 38 & 0.029 & 554 & 0.284 & 1.645 & 1.86 & 0.127 & 1.85 \\
\hline Juniperus flaccida Schltdl. & Árbol* & 43 & 0.042 & 179 & 0.127 & 0.5358 & 0.6061 & 0.143 & 0.597 \\
\hline $\begin{array}{l}\text { Taxodium huegelii C.Lawson } \\
\text { Familia Erythroxylaceae }\end{array}$ & Árbol* & - & 0.000 & 34 & 0.045 & 0.1587 & 0.1795 & 0 & 0.113 \\
\hline $\begin{array}{l}\text { Erythroxylum rotundifolium Lunan } \\
\text { Familia Euphorbiaceae }\end{array}$ & Árbol ${ }^{*}$ & 14 & 0.031 & 22 & 0.021 & 0.1127 & 0.1275 & 0.0467 & 0.0733 \\
\hline Sebastiania pavoniana (Müll.Arg.) Müll.Arg. & Arbusto* & 128 & 0.125 & 154 & 0.139 & 0.7541 & 0.8529 & 0.427 & 0.513 \\
\hline Sapium sp. & Arbusto $^{\star \star}$ & 80 & 0.071 & 107 & 0.103 & 0.6143 & 0.6948 & 0.267 & 0.357 \\
\hline Cnidoscolus tubulosus (Müll. Arg.) I. M. Johnst. & 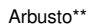 & 31 & 0.029 & 23 & 0.028 & 0.1496 & 0.1692 & 0.103 & 0.0767 \\
\hline $\begin{array}{l}\text { Jatropha sp. } \\
\text { Familia Fabaceae }\end{array}$ & Arbusto** & - & 0.000 & 3 & 0.006 & 0.0089 & 0.0101 & 0 & 0.01 \\
\hline Mimosa polyantha Benth. & 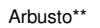 & 1154 & 0.391 & 719 & 0.284 & 4.315 & 4.881 & 3.85 & 2.4 \\
\hline Acacia bilimekii J.F.Macbr. & Arbusto* & 204 & 0.127 & 972 & 0.389 & 2.842 & 3.215 & 0.68 & 3.24 \\
\hline Acacia cochliacantha Willd. & Arbusto** & 335 & 0.169 & 577 & 0.268 & 2.236 & 2.529 & 1.12 & 1.92 \\
\hline Sp. 2 Nanota & 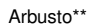 & 481 & 0.136 & 414 & 0.181 & 2.047 & 2.315 & 1.6 & 1.38 \\
\hline Leucaena leucocephala (Lam.) de Wit & Árbol** & 299 & 0.144 & 149 & 0.089 & 1.253 & 1.417 & 0.997 & 0.497 \\
\hline
\end{tabular}


Tabla 1. Continuación

\begin{tabular}{|c|c|c|c|c|c|c|c|c|c|}
\hline Mimosa lacerata Rose & Arbusto** & 90 & 0.065 & 316 & 0.201 & 1.13 & 1.278 & 0.3 & 1.05 \\
\hline Acacia coulteri A.Gray & Arbusto** & 306 & 0.127 & 65 & 0.051 & 1.088 & 1.231 & 1.02 & 0.217 \\
\hline Eysenhardtia polystachya (Ortega) Sarg & Arbusto** & 229 & 0.090 & 104 & 0.068 & 0.9396 & 1.063 & 0.763 & 0.347 \\
\hline Conzattia multiflora (Robinson) Standl. & Árbol * & 218 & 0.090 & 94 & 0.052 & 0.8636 & 0.9768 & 0.727 & 0.313 \\
\hline Senna wislizeni (A.Gray) H.S.Irwin \& Barneby & Arbusto** & 8 & 0.013 & 266 & 0.188 & 0.7895 & 0.893 & 0.0267 & 0.887 \\
\hline Brongniartia intermedia Moric. & Arbusto** & 13 & 0.018 & 276 & 0.188 & 0.7109 & 0.804 & 0.0433 & 0.92 \\
\hline Lysiloma tergemina Benth & Árbol ${ }^{\star}$ & 148 & 0.146 & 13 & 0.022 & 0.4213 & 0.4765 & 0.493 & 0.0433 \\
\hline Mimosa benthamii J.F.Macbr & Arbusto** & 57 & 0.040 & 103 & 0.080 & 0.4026 & 0.4553 & 0.19 & 0.343 \\
\hline Sphinga acatlensis (Benth.) Barneby \& J.W.Grimes & Árbol ${ }^{\star}$ & 57 & 0.050 & 102 & 0.111 & 0.3932 & 0.4448 & 0.190 .34 & \\
\hline Lysiloma divaricatum (Jacq.) J.F.Macbr. & Árbol** & 43 & 0.038 & 23 & 0.022 & 0.251 & 0.2839 & 0.143 & 0.0767 \\
\hline Senna holwayana (Rose) H.S.Irwin \& Barneby & Arbusto** & 60 & 0.040 & 14 & 0.021 & 0.217 & 0.2454 & 0.2 & 0.0467 \\
\hline Styphnolobium burseroides M. Sousa, Rudd \& González Medrano, Francisco & Árbol ${ }^{*}$ & 48 & 0.032 & 20 & 0.021 & 0.2077 & 0.2349 & 0.16 & 0.0667 \\
\hline Acacia farnesiana (L.) Willd. & Arbusto** & - & 0.000 & 84 & 0.176 & 0.1992 & 0.2253 & 0 & 0.28 \\
\hline Senna andrieuxii (Benth.) H.S.Irwin \& Barneby & Árbol ${ }^{\star}$ & 45 & 0.041 & 16 & 0.021 & 0.1745 & 0.1974 & 0.15 & 0.0533 \\
\hline Lysiloma acapulcense (Kunth) Benth. & Árbol* & 53 & 0.033 & 2 & 0.005 & 0.1575 & 0.1781 & 0.177 & 0.0067 \\
\hline Mimosa albida Willd. & Arbusto** & 27 & 0.039 & 5 & 0.009 & 0.1351 & 0.1528 & 0.09 & 0.0167 \\
\hline Prosopis laevigata (Willd.) M.C.Johnst. & Árbol** & 5 & 0.009 & 35 & 0.064 & 0.1301 & 0.1471 & 0.0167 & 0.117 \\
\hline Lonchocarpus caudatus Pittier & Árbol*夫 & 30 & 0.043 & 4 & 0.008 & 0.1194 & 0.135 & 0.1 & 0.0133 \\
\hline Mimosa adenantheroides (M. Martens \& Galeotti) Benth. & Arbusto** $^{\star *}$ & 3 & 0.006 & 28 & 0.044 & 0.0713 & 0.0806 & 0.01 & 0.0933 \\
\hline Acacia Sp1. & Arbusto** & 3 & 0.007 & 20 & 0.031 & 0.0602 & 0.0681 & 0.01 & 0.0667 \\
\hline Hesperalbizia occidentalis (Brandegee) Barneby \& J.W.Grime & Arbusto* & 7 & 0.014 & 11 & 0.014 & 0.0514 & 0.0582 & 0.0233 & 0.0367 \\
\hline Calliandra eriophylla Benth. & Arbusto* & 8 & 0.019 & 11 & 0.028 & 0.0476 & 0.0539 & 0.0267 & 0.0367 \\
\hline Leucaena esculenta (DC.) Benth. & Árbol* & - & 0.000 & 4 & 0.008 & 0.0176 & 0.0199 & 0 & 0.0133 \\
\hline Sp. 1 Garbancillo & Arbusto** & 6 & 0.008 & - & 0.000 & 0.0174 & 0.0197 & 0.02 & 0 \\
\hline Coursetia glandulosa A. Gray & Arbusto* & - & 0.000 & 3 & 0.006 & 0.0141 & 0.0159 & 0 & 0.01 \\
\hline Pithecellobium dulce (Roxb.) Benth & Árbol* & 1 & 0.003 & - & 0.000 & 0.0027 & 0.0031 & 0.0033 & 0 \\
\hline \multicolumn{10}{|l|}{ Familia Fagaceae } \\
\hline $\begin{array}{l}\text { Quercus glaucoides M.Martens \& Galeotti } \\
\text { Quercus magnoliifolia Née }\end{array}$ & Árbol* & 464 & 0.220 & 429 & 0.189 & 2.102 & 2.378 & 1.55 & 1.43 \\
\hline Quercus magnoliifolia Née & Árbol ${ }^{\star}$ & 41 & 0.085 & 241 & 0.206 & 0.6317 & 0.7145 & 0.137 & 0.803 \\
\hline $\begin{array}{l}\text { Quercus acutifolia Née } \\
\text { Familia Fouquieriaceae }\end{array}$ & Árbol ${ }^{\star}$ & 14 & 0.024 & - & 0.000 & 0.0365 & 0.0413 & 0.0467 & 0 \\
\hline \multicolumn{9}{|l|}{ Familia Lamiaceae } & 0.127 \\
\hline Vitex mollis Kunth & Arbusto* & 37 & 0.034 & 7 & 0.013 & 0.1274 & 0.144 & 0.123 & 0.0233 \\
\hline \multicolumn{10}{|l|}{ Familia Malpighiaceae } \\
\hline Lasiocarpus sp. & Arbusto** & 205 & 0.104 & 247 & 0.115 & 1.157 & 1.308 & 0.683 & 0.823 \\
\hline Bunchosia lanceolata Turcz & Arbusto** & 49 & 0.055 & 246 & 0.168 & 0.8096 & 0.9157 & 0.163 & 0.82 \\
\hline Malpighia mexicana A. Juss. & Arbusto* & 113 & 0.051 & 114 & 0.076 & 0.6342 & 0.7174 & 0.377 & 0.38 \\
\hline \multicolumn{10}{|l|}{ Familia Malvaceae } \\
\hline Melochia sp. & & 12 & 0.031 & 385 & 0.228 & 0.9709 & 1.098 & 0.04 & 1.28 \\
\hline Ceiba aesculifolia (H.B.K.) Britten \& Baker & Árbol* & 121 & 0.058 & 51 & 0.029 & 0.5 & 0.5656 & 0.403 & 0.17 \\
\hline Heliocarpus terebinthinaceus (DC.) Hochr. & Arbusto** & 54 & 0.040 & 46 & 0.042 & 0.3198 & 0.3617 & 0.18 & 0.153 \\
\hline Ceiba parvifolia Rose & Árbol ${ }^{\star}$ & 56 & 0.040 & 24 & 0.022 & 0.2431 & 0.2749 & 0.187 & 0.08 \\
\hline $\begin{array}{l}\text { Guazuma ulmifolia Lam. } \\
\text { Familia Meliaceae }\end{array}$ & Árbol** & 4 & 0.011 & - & 0.000 & 0.0151 & 0.0171 & 0.0133 & 0 \\
\hline $\begin{array}{l}\text { Cedrela salvadorensis Standl. } \\
\text { Familia Moraceae }\end{array}$ & \multicolumn{4}{|c|}{ Familia Moraceae } & 0.011 & 0.0379 & 0.0428 & 0.0067 & 0.0267 \\
\hline Ficus cotinifolia Kunth & Árbol** & 8 & 0.009 & 1 & 0.003 & 0.0274 & 0.031 & 0.0267 & 0.0033 \\
\hline Ficus petiolaris Kunth & Árbol** & - & 0.000 & 4 & 0.007 & 0.0159 & 0.018 & 0 & 0.0133 \\
\hline \multicolumn{10}{|l|}{ Familia Oleaceae } \\
\hline $\begin{array}{l}\text { Forestiera angustifolia Torr. } \\
\text { Familia Onagraceae }\end{array}$ & Arbusto** & 517 & 0.247 & 225 & 0.149 & 2.282 & 2.581 & 1.72 & 0.75 \\
\hline \multicolumn{9}{|l|}{ Familia Opiliaceae } & 0.07 \\
\hline Agonandra obtusifolia Standl. & Árbol* & 57 & 0.037 & 93 & 0.073 & 0.4041 & 0.4571 & 0.19 & 0.31 \\
\hline \multicolumn{10}{|l|}{ Familia Rhamnaceae } \\
\hline Colubrina triflora Brongn.ex Sweet & Arbusto* & 272 & 0.128 & 201 & 0.111 & 1.319 & 1.492 & 0.907 & 0.67 \\
\hline Karwinskia mollis Schltdl. & Arbusto* & 69 & 0.054 & 172 & 0.104 & 0.681 & 0.7703 & 0.23 & 0.573 \\
\hline Rhamnus humboldtiana Willd. ex Schult. & Arbusto* & - & 0.000 & 29 & 0.027 & 0.1016 & 0.115 & 0 & 0.0967 \\
\hline Ceanothus caeruleus Lag. & Arbusto** & - & 0.000 & 23 & 0.026 & 0.0556 & 0.063 & 0 & 0.0767 \\
\hline $\begin{array}{l}\text { Ziziphus amole (Sessé \& Moc.) M. C. Johnst. } \\
\text { Familia Rubiaceae }\end{array}$ & Árbol ${ }^{\star}$ & 1 & 0.003 & - & 0.000 & 0.0042 & 0.0048 & 0.0033 & 0 \\
\hline Hintonia latiflora (Sessé \& Moc. Ex. DC.) Bullock & Árbol ${ }^{\star}$ & 83 & 0.060 & 31 & 0.024 & 0.3636 & 0.4112 & 0.277 & 0.103 \\
\hline Randia armata (Sw.) DC. & Arbusto* & 38 & 0.040 & - & 0.000 & 0.1071 & 0.1212 & 0.127 & 0 \\
\hline Randia thurberi S. Watson & Arbusto* & - & 0.000 & 1 & 0.003 & 0.0037 & 0.0042 & 0 & 0.0033 \\
\hline Familia Rutaceae & & & & & & & & & \\
\hline Ptelea trifoliata L. & Arbusto* & 197 & 0.116 & 125 & 0.098 & 1.045 & 1.182 & 0.657 & 0.417 \\
\hline Zanthoxylum ciliatum Engl. & Arbusto* & 28 & 0.043 & 16 & 0.019 & 0.1328 & 0.1502 & 0.0933 & 0.0533 \\
\hline Familia Salicaceae & & & & & & & & & \\
\hline Casearia corymbosa Kunth & Arbusto** & 58 & 0.049 & 7 & 0.010 & 0.2489 & 0.2816 & 0.193 & 0.0233 \\
\hline Xylosma intermedia (Seem.) Griseb. & Árbol** & 22 & 0.026 & 12 & 0.025 & 0.0868 & 0.0982 & 0.0733 & 0.04 \\
\hline Neopringlea viscosa (Liebm.) Rose & Arbusto** & 9 & 0.011 & - & 0.000 & 0.0244 & 0.0276 & 0.03 & 0 \\
\hline Familia Sapindaceae & & & & & & & & & \\
\hline $\begin{array}{l}\text { Dodonaea viscosa (L.)Jacq. } \\
\text { Familia Sapotaceae }\end{array}$ & Arbusto** & 796 & 0.396 & 1649 & 0.694 & 5.538 & 6.264 & 2.65 & 5.5 \\
\hline Sideroxylon palmeri (Rose) T.D.Penn. & Árbol* & 71 & 0.091 & - & 0.000 & 0.2045 & 0.2313 & 0.2363 & 0 \\
\hline Sp sin identificar & & & & & & & & & \\
\hline Sp. 2 & & 155 & 0.074 & 142 & 0.088 & 0.8494 & 0.9607 & 0.517 & 0.473 \\
\hline Sp. 1 & & 85 & 0.049 & 49 & 0.045 & 0.7524 & 0.851 & 0.0001 & 0.163 \\
\hline Sp. 3 & & 53 & 0.058 & 22 & 0.028 & 0.2016 & 0.228 & 0.177 & 0.0733 \\
\hline
\end{tabular}




\begin{tabular}{|c|c|c|c|c|c|c|c|c|c|}
\hline Sp. 4 & & - & 0.000 & 50 & 0.167 & 0.1545 & 0.1748 & 0 & 0.167 \\
\hline sp. 7 & & 24 & 0.026 & 38 & 0.032 & 0.1492 & 0.1688 & 0.08 & 0.127 \\
\hline Sp. 5 & & 25 & 0.037 & 7 & 0.020 & 0.1122 & 0.1269 & 0.0833 & 0.0233 \\
\hline Sp. 6 & & - & 0.000 & 20 & 0.032 & 0.0756 & 0.0855 & 0 & 0.0667 \\
\hline Familia Verbenaceae & & & & & & & & & \\
\hline $\begin{array}{l}\text { Lippia graveolens Kunth } \\
\text { Familia Zygophyllaceae }\end{array}$ & Arbusto ${ }^{\star \star}$ & 250 & 0.210 & 1022 & 0.425 & 3.126 & 3.536 & 0.833 & 3.41 \\
\hline Sp. 1 & & 29 & 0.028 & 8 & 0.010 & 0.1139 & 0.1289 & 0.0967 & 0.0267 \\
\hline
\end{tabular}

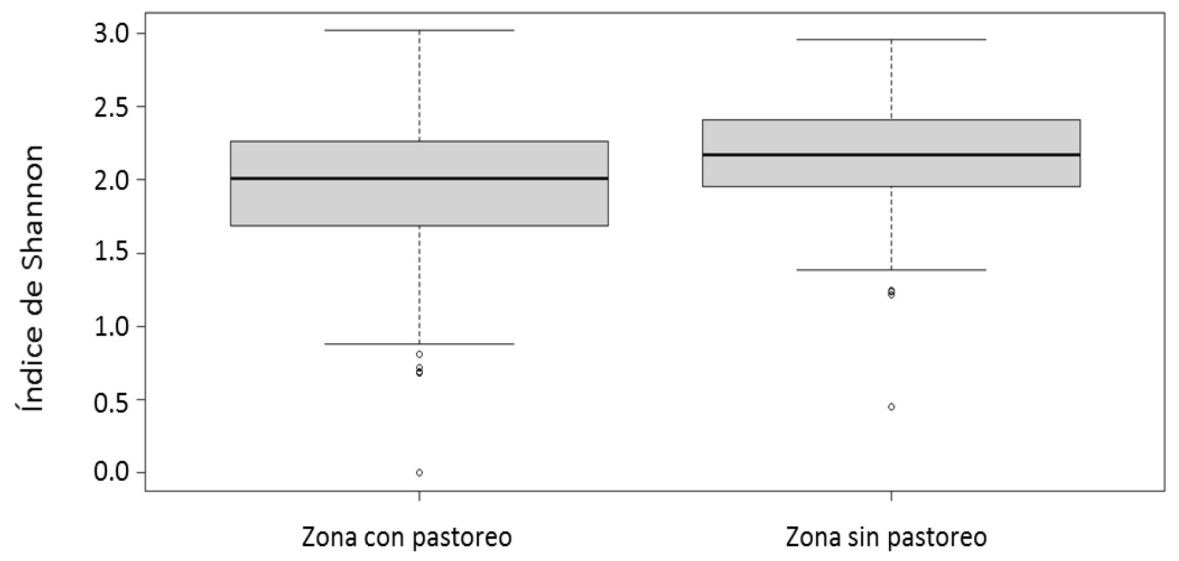

Figura 2. Valores de Índice de Shannon-Weiner utilizando la prueba de "t" modificada por Hutcheson.

\section{DISCUSIÓN}

La pérdida de biodiversidad es un asunto de suma importancia debido a la presión que se tiene sobre los recursos naturales. En la REST las actividades agropecuarias, así como la extracción de recursos, han favorecido el establecimiento de especies secundarias indicadoras de degradación del ecosistema. A pesar de esto, actualmente la REST presenta gran diversidad de árboles y arbustos (131 especies) en las zonas muestreadas comparado a lo reportado por otras investigaciones en la selva baja caducifolia, en áreas de muestreo entre 0.1 a 1 ha, que indican un rango de 111 a 148 especies (en zonas no pastoreadas). Por ejemplo, Gallardo-Cruz et al. (2005), en Cerro Verde Nizanda, Oaxaca, reportaron 145 especies leñosas (en zonas donde no había indicio de perturbación humana); Durán et al. (2006) obtuvieron 148 especies en la zona de Chamela, Jalisco; Gordon y Newton (2006) reportaron 111 especies en 0.1 ha, y 132 especies en 0.4 ha en Huatulco, Oaxaca; mientras que Williams-Linera y Lorea (2009), reportan 122 especies leñosas en Paso de Ovejas, Veracruz.
En la REST la familia más abundante con 31 especies coincide con lo reportado por Rzedowski (2006) y Casanova-Lugo et al. (2014) quienes encontraron que, de las angiospermas en la selva baja caducifolia, la familia más representativa es Fabaceae (Leguminosae), tanto en número de especies como en individuos, así como en importancia y dominancia de los estratos arbóreos. También concuerda con lo reportado por Villanueva-Partida et al. (2019) para zonas de pastoreo de ganado bovino con árboles dispersos en potreros en la Sierra Sur del estado de Tabasco (México). En la REST la riqueza y diversidad de especies arbóreas y arbustivas en zonas pastoreadas y zonas sin pastoreo es alta de acuerdo a lo obtenido mediante la curva de acumulación de especies, así como, por el Índice de ShannonWiener $\left(H^{\prime}\right)$ cuyos resultados mostraron diferencias significativas entre zonas; así mismo se encontraron diferencias entre la cantidad de individuos dentro de especies. Al respecto, McEvoy et al. (2006) estudiaron bosques de Irlanda y Al-Rowaily et al. (2015) pastizales degradados del oeste de Arabia Saudita; en sitios pastoreados y sin pastoreo, en- 

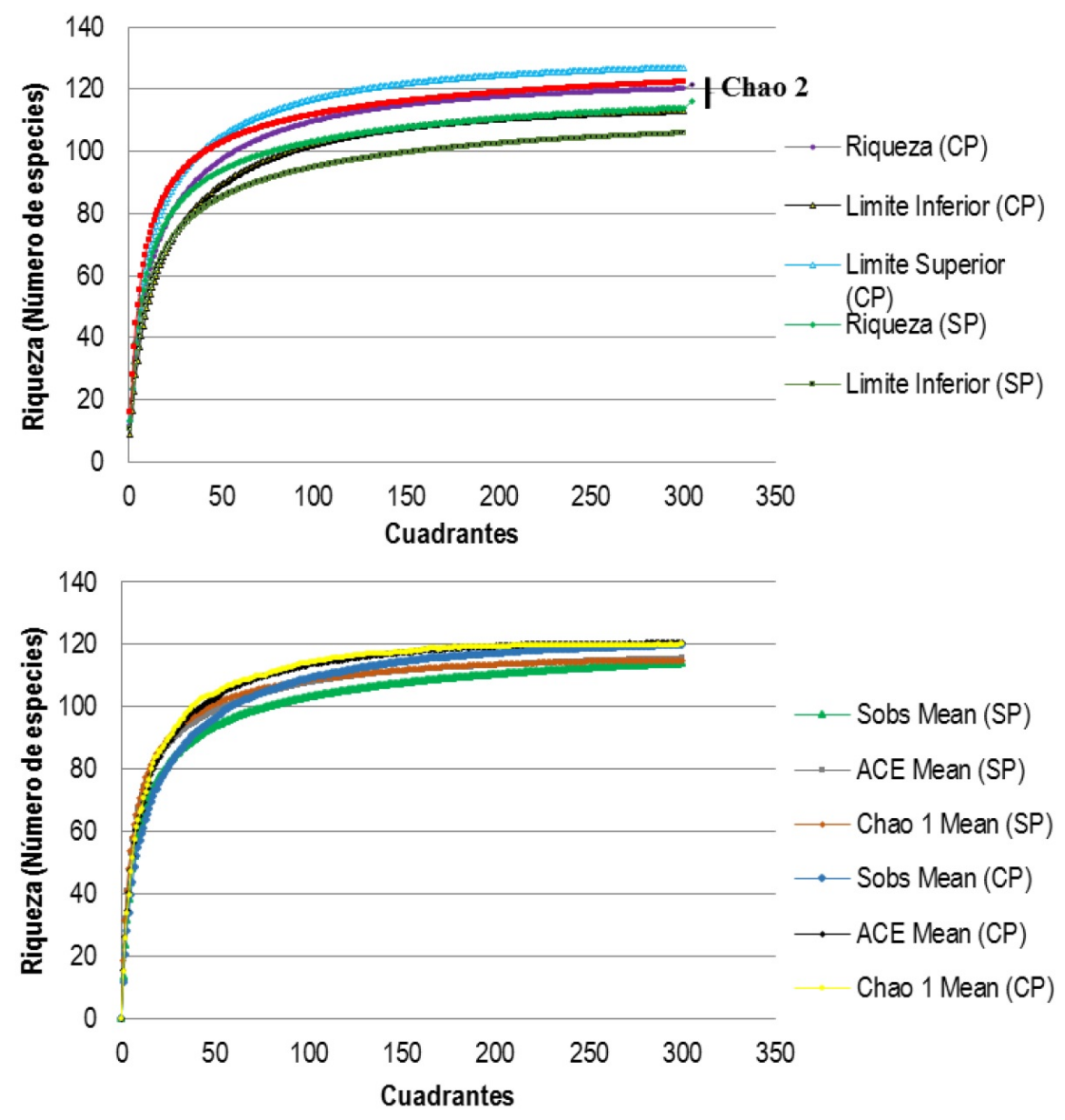

Figura 3. Curva de acumulación de especies (Riqueza) con varios índices.

contraron que las zonas restringidas de pastoreo presentan mayor riqueza de especies. Las zonas pastoreadas, por su parte, presentan un gran porcentaje de especies no palatables. Por otro lado, Hernández et al. (2000), mencionaron que, en los bosques de pino, bosque de pino-encino y bosque mesófilo de montaña, con diferencias en la composición de árboles jóvenes en zonas pastoreadas y sin pastoreo; además, en el bosque de pino también se halló un menor número de especies. Encontrando diferencias significativas en el número de especies para los tres tipos de bosque; sin embargo, entre sitios restringidos y con pastoreo no reportaron diferencias significativas.

Una de las especies dominantes en la zona con pastoreo fue Opuntia depressa, especie endémica de México que se distribuye en la cuenca del Balsas (Fernández-Nava et al. 1998), que, por su gran éxito reproductivo y tolerancia a perturbaciones, es muy abundante. Esta especie se desarrolla en bosques tropicales de hoja caduca, matorral xerófilo y pastizales secundarios (Arias et al. 2013). Especies como Ipomoea pauciflora, Guazuma ulmifolia, Acacia cochliacantha, A. farnesiana, Mimosa polyantha, Senna skinneri, Pithecellobium acatlense se consideran de vegetación secundaria (Arias et al. 2002, Contreras-MacBeath et al. 2002) y son indicadoras de sucesión y por tanto de ocurrencia de disturbio. De acuerdo a lo reportado por Arias et al. (2013) con base a la especie O. depressa, y otras que presentaron un gran número de individuos como Brahea dulcis (Kunth) Mart. y Dodonaea viscosa (L.), así 
como a la ausencia de especies típicas de la selva baja como Stenocerueus stellatus Pfeiffer, se tienen indicios que la REST actualmente enfrenta un cierto deterioro tanto en las zonas pastoreadas como las no pastoreadas.

Son múltiples los efectos que se generan en las selvas a partir del pastoreo. Al respecto, Baraza y Valiente-Banuet (2012), señalan que el ramoneo puede ser positivo para algunas especies, ya que genera compensación en el crecimiento, la producción de flores, frutos $y / 0$ semillas respecto a plantas no consumidas. Ellos aluden que, al restringir las plantas del pastoreo, se presenta mayor cobertura, y que algunas especies muestran una disminución en la inversión reproductiva, lo que sugiere que están adaptadas al ramoneo. Por lo que restringir el pastoreo puede ser negativo para algunas especies palatables y crucial para otras. Por otro lado, Bugalho et al. (2011) señalan que muchas especies se han adaptado a la condición de pastoreo y exhiben gran capacidad de rebrote y de letargo como el caso de arbustivas y especies herbáceas; por lo que, disminuir la carga impacta de forma negativa la regeneración de ciertas especies como las del género Quercus. También se ha reportado que el pastoreo afecta la diversidad, Fischer y Wipf (2002) en su estudio realizado en pastos subalpinos encontraron que el pastoreo es nocivo porque condiciona la riqueza y calidad agrícola, pues para mantener los prados subalpinos debe mantenerse el segado, lo que contribuye de forma negativa a la biodiversidad, ocasionando pérdida de especies.

La curva de acumulación de especies en la presente investigación mostró una asíntota, lo que indica que se registró con alto grado de probabilidad la diversidad de las comunidades, los intervalos se solapan con el valor máximo de riqueza de especies, lo que implica que no hay diferencias significativas entre la riqueza observada y la esperada en cada zona, confirmando que el muestreo fue extensivo, exhaustivo y suficiente para capturar la diversidad de las zonas con y sin pastoreo.

El valor del índice de Shannon alcanzado en la REST para zonas con pastoreo y sin pastoreo (3.72 y 3.88 , respectivamente) se consideran altos y se encuentran en el rango que presentaron WilliamsLinera y Lorea (2009) de 3.76, así como el de Durán et al. (2006) de 3.08. Lo que indica que ambas zonas presentan gran diversidad arbórea-arbustiva. A pesar de que la presión de uso en esta selva es muy alta se sigue conservando una importante diversidad arbórea-arbustiva, no obstante, se requieren hacer estudios para conocer si existe substitución de especies. Las actividades como la ganadería en los bosques y selvas han convertido a los diversos tipos de vegetación en remanente de bosque, incluso los han transformado en bosques y selvas secundarios, que actualmente siguen siendo alterados e invadidos por flora y fauna exótica (Arévalo et al. 2007), lo que conlleva a la pérdida de especies nativas, aspecto que también requiere estudiarse en el área muestreada pues no se encontraron pocos individuos de especies típicas como las de los géneros Bursera, Amphipterygium y Ceiba, entre otras (FernándezNava et al. 1998).

\section{CONCLUSIONES}

La riqueza y diversidad de flora arbóreoarbustiva de acuerdo con los índices utilizados difirió entre las zonas pastoreadas y las zonas sin pastoreo; cambiando la composición botánica al encontrarse que el número de individuos por especie fue diferente en cada zona. El valor del Índice de Shannon-Wiener $\left(H^{\prime}\right)$ para las zonas con y sin pastoreo es alto y se encuentra dentro de los límites reportados por otros autores. Muchas de las especies reportadas, corresponden a sucesión secundaria e indicadoras de disturbio y su número de individuos es más elevado que las especies nativas. Por lo que, se sugiere que aun cuando el pastoreo puede modificar la diversidad, no es el único elemento que está incidiendo ya que hay otras actividades agrícolas y de subsistencia en los ecosistemas que pueden influenciar. Debido a ello, la riqueza y diversidad pueden ser altas, pero no siempre se da con las especies originales, lo que puede modificar la estructura y funcionalidad de la diversidad arbóreo-arbustiva nativa. 


\section{AGRADECIMIENTOS}

Al Consejo Nacional de Ciencia y Tecnología (CONACyT) por la beca recibida por la primera autora. A la gente de las comunidades de San Agustín
Ahuehuetla, Colonia Agrícola Hidalgo, San Miguel Atlapulco, San Juan Huiluco y Santa Ana Coatepec, Puebla por las facilidades que dieron en la realización de este estudio.

\section{LITERATURA CITADA}

Al-Rowaily SL, El-Bana MI, Al-Bakre DA, Assaeed AM, Hegazy AK, Ali MB (2015) Effects of open grazing and livestock exclusion on floristic composition and diversity in natural ecosystem of Western Saudi Arabia. Saudi Journal of Biological Sciences 22: 430-437.

Arévalo JR, Chinea E, Barquín E (2007) Pasture management under goat grazing on Canary Islands. Agriculture, Ecosystems and Environment 118: 291-296.

Arévalo JR, De Nascimento L, Fernández-Lugo S, Mata J, Bermejo L (2011) Grazing effects on species composition in different vegetation types (La Palma, Canary Islands). Acta Oecologica 37: 230-238.

Arias D, Dorado O, Maldonado B (2002) Biodiversidad e importancia de la selva baja caducifolia de la Reserva de la Biosfera Sierra de Huautla. Biodiversitas 45: 7-12.

Arias S, Hernández C, Valverde T, Zavala-Hurtado A (2013) Opuntia depressa. The IUCN Red List of Threatened Species 2013: e.T152573A652831. http://dx.doi.org/10.2305/IUCN.UK.2013-1.RLTS.T152573A652831.en.

Baraza E, Valiente-Banuet A (2008) Seed dispersal by domestic goats in a semiarid thornscrub of Mexico. Journal of Arid Environments 72: 1973-1976.

Baraza E, Valiente-Banuet A (2012) Efecto de la exclusión de ganado en dos especies palatables del matorral xerófilo del Valle de Tehuacán, México. Revista Mexicana de Biodiversidad 83: 1145-1151.

Belsky AJ, Blumenthal DM (1997) Effects of livestock grazing on stand dynamics and soils in upland forests of the interior west. Conservation Biology 11: 315-327.

Benthien O, Braun M, Riemann JC, Stolter C (2018) Long-term effect of sheep and goat grazing on plant diversity in a semi-natural dry grassland habitat. Heliyon 4: 1-17.

Bermejo LA, De Nascimento L, Mata J, Fernández-Lugo S, Camacho A, Arévalo JR (2012) Responses of plant functional groups in grazed and abandoned areas of a Natural Protected Area. Basic and Applied Ecology 13: 312-318.

Bugalho MN, Lecomte X, Gonçalves M, Caldeira MC, Branco M (2011) Establishing grazing and grazing-excluded patches increases plant and invertebrate diversity in a Mediterranean oak woodland. Forest Ecology and Management 261: 2133-2139.

Casanova-Lugo F, González-Gómez J, Flores-Estrada M, López-Santiago G, García-Gómez M (2014) Estructura, composición y usos de los árboles de la selva baja caducifolia en Apatzingán, Michoacán. Tropical and Subtropical Agroecosystems 17: 255-259.

Cavieres LA, Badano El (2009) Do facilitative interactions increase species richness at the entire community level? Journal of Ecology 97: 1181-1191.

Chao A, Chazdon RL, Colwell RK, Shen TJ (2005) A new statistical approach for assessing similarity of species composition with incidence and abundance data. Ecology Letters 8: 148-159.

Colwell RK (2005) EstimateS 8.2 User's Guide. Department of Ecology \& Evolutionary Biology, University of Connecticut, Storrs. USA. http://purl.oclc.org/estimates. Fecha de consulta: 19 de abril de 2020. 
Colwell RK, Mao CX, Chang J (2004) Interpolating, extrapolating, and comparing incidence-based species accumulation curves. Ecology 85: 2717-2727.

Contreras-MacBeath T, Ongay-Delhumeau E, Sorani DV (2002) Programa Estatal de Ordenamiento Territorial Sustentable de Morelos Fases I, II y III. Incluyendo los subsistemas Natural, Social y Económico. SEDESOL. México. 600p.

Díaz S, Lavorel S, Mclntyre S, Falczuk V, Casanoves F, Milchunas DG Skarpe C, Rusch G, Zhang W, Clark H, Campbell BD (2007) Plant traits responses to grazing: a global synthesis. Global Change Biology 13: 313-34.

Durán E, Meave J, Lott E, Segura G (2006) Structure and tree diversity patterns at the landscape level in a Mexican tropical deciduous forest. Boletín de la Sociedad Botánica de México 79: 43-60.

Fernández-Nava R, Rodríguez-Jiménez C, Arreguín-Sánchez ML, Rodríguez-Jiménez A (1998) Listado florístico de la cuenca del río Balsas, México. Polibotánica 9: 1-151.

Fischer M, Wipf S (2002) Effect of low-intensity grazing on the species-rich vegetation of traditionally mown subalpine meadows. Biological Conservation 104: 1-11.

Gallardo-Cruz JA, Meave JA, Pérez EA (2005) Estructura, composición y diversidad de la selva baja caducifolia del Cerro Verde, Nizanda (Oaxaca), México. Boletín de la Sociedad Botánica de México 76: 19-35.

García E (2004) Modificaciones del sistema de clasificación climática de Köppen (para adaptarlo a las condiciones de la República Mexicana). $5^{a}$ Edición. Instituto de Geografía, UNAM. México. 90p.

García RR, Celaya R, García U, Osoro K (2012) Goat grazing, its interactions with other herbivores and biodiversity conservation issues. Small Ruminant Research 107: 49-64.

García-Romero A, Mendoza-Robles KI, Galicia-Sarmiento L (2005) Valoración de la selva baja caducifolia en la cuenca del río Papagayo (Guerrero), México. Investigaciones Geográficas, Boletín del Instituto de Geografía, UNAM 56: 77-100.

Gordon JE, Newton AC (2006) The potential misapplication of rapid plant diversity assessment in tropical conservation. Journal for Nature Conservation 14: 117-126.

Hammer O, Harper DAT, Ryan PD (2001) PAST: Paleontological statistics software package for education and data analysis. Palaeontologia Electronica 4: 1-9.

Hernández G, Sánchez LR, Carmona TF, Pineda MR, Cuevas R (2000) Efecto de la ganadería extensiva sobre la regeneración arbórea de los bosques de la Sierra de Manantlán. Madera y Bosques 6: 13-28.

Huerta-Zamacona S, Vidal-Corona A, Rodríguez-Rodríguez J, Bonilla B M, Mora-Pérez M, Vázquez-Martínez S, Ortiz-y-Rivera GA, Bravo-Hernández F (2009) Principales árboles y arbustos en la selva baja caducifolia de la Mixteca Baja poblana, Puebla, México. Primera edición. Fundación Produce Puebla. México. 199p.

INEGI (2017) Anuario estadístico y geográfico de Puebla 2017. Instituto Nacional de Estadística y Geografía, Gobierno del Estado de Puebla, México. 943p.

Kalacska M, Sanchez-Azofeifa GA, Calvo-Alvarado JC, Quesada M, Rivard B, Janzend DH (2004) Species composition, similarity and diversity in three successional stages of a seasonally dry tropical forest. Forest Ecology and Management 200: 227-247.

Kauffman JB, Steele MD, Cummings DL, Jaramillo VJ (2003) Biomass dynamics associated with deforestation, fire, and, conversion to cattle pasture in a Mexican tropical dry forest. Forest Ecology and Management 176: $1-12$. 
Magurran AE (1988) Ecological diversity and its measurement. Princeton University Press, New Jersey. 179p.

McEvoy PM, Flexen M, McAdam JH (2006) The effects of livestock grazing on ground flora in broadleaf woodlands in Northern Ireland. Forest Ecology and Management 225: 39-50.

Müller JL, Babu MM, Saklani PL, Mayer AC, Marquardt S, Kreuzer M (2011) Forage resource use by cattle or goats at an Indian protected area: Differences and implications for conservation. Journal of Arid Environments 77: 130-137.

Nautiyal S, Kaechele H (2006) Adverse impacts of pasture abandonment in Himalayan protected areas: Testing the efficiency of a Natural Resource Management Plan (NRMP). Environmental Impact Assessment Review 27: 109-125.

Pla L (2006) Biodiversidad: inferencia basada en el índice de Shannon y la riqueza. Interciencia 31: 583-590.

Rodríguez-Acosta M, Coombes AJ, Jiménez-Ramírez J (2009). Plantas silvestres de Puebla. Herbario y Jardín Botánico BUAP. Benemérita Universidad Autónoma de Puebla. México. 235p.

Rzedowski J (2006) Bosque tropical caducifolio: En: Comisión Nacional para el Conocimiento y Uso de la Biodiversidad (Ed.). Vegetación de México. 1ra. Edición digital. México. pp: 200-214.

SEMARNAP (1998) Mapa de suelos dominantes de la República Mexicana. (Primera aproximación 1998). Escala 1:4 000 000. Subsecretaría de Recursos Naturales, México.

SMADSOT (2011) Estudio previo justificativo para la declaratoria de la Reserva Estatal "SIERRA DEL TENTZO". Secretaría de Medio Ambiente, Desarrollo Sustentable y Ordenamiento Territorial, Gobierno del Estado de Puebla. Puebla, México. 46p.

SSAOT (2011a) Decreto de la Reserva Estatal Sierra del Tentzo como Área Natural Protegida. Secretaria de Sustentabilidad Ambiental y Ordenamiento Territorial. Periódico Oficial del estado de Puebla. México. 12p.

SSAOT (2011b) Programa de manejo del Área Natural Protegida, Reserva Estatal Sierra del Tentzo. Secretaria de Sustentabilidad Ambiental y Ordenamiento Territorial. Periódico Oficial del estado de Puebla. México. $13 p$.

Trejo I, Dirzo R (2000) Deforestation of seasonally dry tropical forest: a national and local analysis in Mexico. Biological Conservation 94: 133-142.

Vedeld P, Jumane A, Wapalila G, Songorwaark A (2012) Protected areas, poverty and conflicts: A livelihood case study of Mikumi National Park, Tanzania. Forest Policy and Economics 21: 20-31.

Villanueva-Partida CR, Casanova-Lugo F, González-Valdivia NA, Villanueva-López G, Oros-Ortega I, Cetzal-Ix W, Basu S (2019) Traditional uses of dispersed trees in the pastures of the mountainous region of Tabasco, Mexico. Agroforestry Systems 93: 383-394.

Villarreal H, Álvarez M, Córdoba S, Escobar F, Fagua G, Gast F, Mendoza H, Ospina M, Umaña AN, Villarreal H (2006) Manual de métodos para el desarrollo de inventarios de biodiversidad. Programa de Inventarios de Biodiversidad. Segunda edición. Instituto de Investigación de Recursos Biológicos Alexander von Humboldt. Bogotá, Colombia. 236p.

Williams-Linera G, Lorea F (2009) Tree species diversity driven by environmental and anthropogenic factors in tropical dry forest fragments of central Veracruz, Mexico. Biodiversity and Conservation 18: 3269-3293. 